\title{
Slow-Fast Duffing Neural Mass Model
}

\author{
Amirhossein Jafarian ${ }^{1, \dagger}$, Dean R. Freestone ${ }^{2,3}$, Dragan Nešić ${ }^{4}$, David B. Grayden ${ }^{3,5}$
}

\begin{abstract}
Epileptic seizures may be initiated by random neuronal fluctuations and/or by pathological slow regulatory dynamics of ion currents. This paper presents extensions to the Jansen and Rit neural mass model (JRNMM) to replicate paroxysmal transitions in intracranial electroencephalogram (iEEG) recordings. First, the Duffing NMM (DNMM) is introduced to emulate stochastic generators of seizures. The DNMM is constructed by applying perturbations to linear models of synaptic transmission in each neural population of the JRNMM. Then, the slow-fast DNMM is introduced by considering slow dynamics (relative to membrane potential and firing rate) of some internal parameters of the DNMM to replicate pathological evolution of ion currents. Through simulation, it is illustrated that the slow-fast DNMM exhibits transitions to and from seizures with etiologies that are linked either to random input fluctuations or pathological evolution of slow states. Estimation and optimization of a log likelihood function (LLF) using a continuous-discrete unscented Kalman filter (CD-UKF) and a genetic algorithm (GA) are performed to capture dynamics of iEEG data with paroxysmal transitions.
\end{abstract}

\section{INTRODUCTION}

Epilepsy is the second most common brain disease, characterized by recurring seizures as a result of abnormal hypersynchronous neuronal activity. At the onset of an epileptic seizure, only a localized region of the brain may be affected (called focal seizures) or all brain regions simultaneously (called generalized seizures). Initiation and termination of epileptic seizures may be caused by random neural fluctuations and/or by pathological slow dynamics of ion currents [1], [6]. Models that provide insights into these etiologies have the potential to enhance treatment and monitoring of patients with epilepsy.

Neural mass models (NMMs) are lumped, biologically informed model of a cortical column that can simulate iEEG recordings [2], [11]. Because epilepsy is a disease associated with pathological large-scale neural activity, NMMs are extensively used to provide insights into underlying generators of this brain pathology. NMMs emulate iEEG data via interactions between mean electrical activities of two or more neuronal populations, each of which is characterized by the dynamics of its mean membrane potential and firing rate [2]. In particular, NMMs have been introduced to replicate

\footnotetext{
*This work was funded by the ARC (Linkage Project LP100200571).

${ }^{1}$ The Wellcome Center For Human Neuroimaging, University Collage London, WC1N 3AR, United Kingdom

${ }^{2}$ Dept. Medicine, The University of Melbourne, VIC 3010, Australia

${ }^{3}$ Seer Medical, 278 Queensberry St, Melbourne, VIC 3000, Australia

${ }^{4}$ Dept. Electrical \& Electronic Engineering, The University of Melbourne, VIC 3010, Australia

${ }^{5}$ Dept. Biomedical Engineering, The University of Melbourne, VIC 3010, Australia

†a.jafarian@ucl.ac.uk
}

spontaneous initiation and termination of seizures. For example, random generators of absence seizures were given by a noise driven thalamocortical NMM [3]. Kalitzin et al. [4] also developed a mathematical model that can replicate stochastic mechanisms of focal seizures. Recently, NMMs have been constructed that link the causation of paroxysmal activities to pathological slow dynamics of internal model parameters [5], [6].

In this paper, slow-fast Duffing NMMs are proposed to replicate both stochastic and quasi-deterministic mechanisms of seizures in iEEG data. These models can provide insights into the pathological dynamics of an isolated cortical column in developing epileptic seizures. The Duffing NMM (DNMM) is constructed by extending the model of synaptic transmission in the JRNMM using Duffing equations. Duffing equations have been used to replicate mean collective dynamics of neuronal populations when they exhibit synchronize activities [7], [8]. Here, we will illustrate that the DNMM can emulate stochastic generators of seizurelike activities. Mathematically, the Duffing equations can be approximated by a linear time-varying system [9], [10] Therefore, the DNMM can be understood approximately as a JRNMM with time-varying parameters. Finally, the slow-fast Duffing NMM is developed by considering slow dynamics for some internal parameters of the DNMM to replicate seizures that are caused either by random input fluctuation and/or pathological slow state dynamics.

In order to be useful for diagnosis, management, and treatment of seizures, a model of epileptic seizures must be able to be assimilated with patient-specific data. In this paper, model identification based on estimation and optimization of a log-likelihood function (LLF) with respect to unknown parameters of the DNMM is used to investigate underlying generators of iEEG with paroxysmal transitions. Optimization of the LLF is performed using a genetic algorithm (GA). Calculation of the LLF (for given model parameters) requires the estimation of hidden states, which is carried out using a continuous-discrete unscented Kalman filter (CD-UKF) [15]. The CD-UKF is used to estimate hidden states of the model because it does not requires model linearization (e.g.,[11]) and also is not sensitive to sampling rate of recorded iEEG data [15].

This paper has four sections. Section II (Methods) describes the slow-fast Duffing NMM and model identification. In Section III, model simulations and identification results are presented. Finally, the conclusions are given in Section IV. 


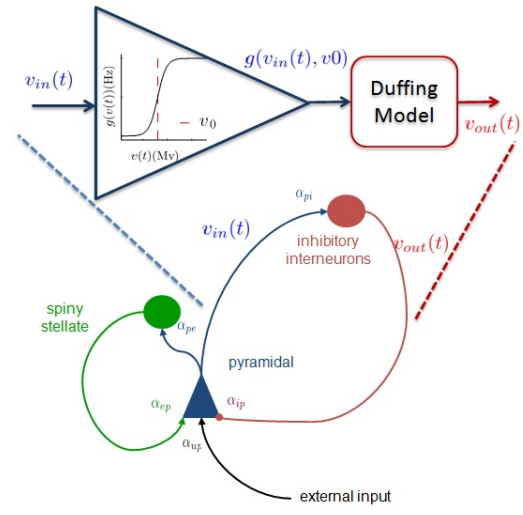

Fig. 1. Duffing Neural Mass Model (DNMM). A post-synaptic potential, $v_{*}(t)(*=p, i, e)$, received by each population is converted to mean firing rate by sigmoid function $g($.$) . The ensuing firing rate is then converted$ to post-synaptic potential by a Duffing equation as a model of synaptic transmission. Connectivity constants are denoted by $\alpha_{k j}(k \neq j=p, i, e)$. Simulated output iEEG is the membrane potential of the pyramidal population.

\section{Material \& Method}

\section{A. Duffing Neural Mass Model}

The Duffing Neural Mass Model (DNMM) is introduced as a generalized JRNMM. The JRNMM models interactions between pyramidal $(p)$ neurons, excitatory stellate $(e)$ and inhibitory $(i)$ interneurons [2]. The DNMM is constructed by applying perturbations to linear models of synaptic transmission in the JRNMM, as shown in Figure 1. In each neuronal population of the DNMM, a postsynaptic potential, $v_{i n}(t)$, is converted to a firing rate by the sigmoid transformation, $g\left(v_{\text {in }}, v_{0}\right)$ :

$$
g\left(v_{\text {in }}(t), v_{0}\right)=\frac{2 e_{0}}{\left(1+e^{\zeta\left(v_{0}-v_{i n}(t)\right)}\right)},
$$

where $v_{0}$ is the firing threshold (at which the postsynaptic potential (PSP) achieves $50 \%$ of the maximum firing rate), $\varsigma$ is the slope at the firing threshold, and $2 e_{0}$ is the maximum firing rate of the neural population. In the DNMM, the dynamics of synaptic transmission is governed by a Duffing equation. The input-to-output conversion of presynaptic firing rates, $g\left(v_{i n}(t), v_{0}\right)$ to postsynaptic potential, $v_{o}(t)(o=p, e, i)$ obeys the differential equation:

$$
\ddot{v}_{o}(t)+2 \zeta \dot{v}_{o}(t)+\zeta^{2}\left(v_{o}(t) \pm \varepsilon v_{o}(t)^{3}\right)=\alpha \zeta g\left(v_{i n}(t), v_{0}\right)
$$

where $0<\varepsilon \ll 1$ is the Duffing stiffness [7], $\zeta$ is synaptic time constant, and $\alpha$ is the gain. By setting $\varepsilon=0$, the linear model of synaptic transmission, as defined by Jansen and Rit [2] can be recovered. Equation (2) can be written in statespace canonical form by defining intermediate states, $v_{j}^{*}(t)$ $(*=p, i, e)$, as:

$$
\begin{aligned}
& \dot{v}_{o}(t)=v_{j}^{*}(t) \\
& \dot{v}_{j}^{*}(t)=-2 \zeta v_{j}^{o}(t)-\zeta^{2}\left(v_{o}(t) \pm \varepsilon v_{o}(t)^{3}\right)+\alpha \zeta g\left(v_{i n}(t), v_{0}\right) .
\end{aligned}
$$

For $\varepsilon>0$, equation (3) is called a "hard Duffing equation"; and for $\varepsilon<0$, it is called a "soft Duffing equation" [7].
In response to external inputs changes, the hard/soft Duffing equation shows progressive stiffness/weakness, which is used to emulate changes in properties of synaptic transmissions in the DNMM.

\section{B. Slow-fast Duffing Neural Mass Model}

In the brain, slow dynamics of ion currents regulate fast activity of membrane potentials and firing rates (activitydepended homeostasis). Pathological dynamics of ion currents may give rise to initiation and termination of seizures [13], [12]. To emulate this effect using mesoscopic models, slow dynamics have been defined for some model parameters in a NMM [5], where a model of spike-frequency adaptation changed of the excitability level of a neuron population using negative feedback from firing rate levels to the value of firing threshold. In the current work, the slow-fast Duffing NMM is developed by considering slow dynamics for $v_{0}$ to model spike-frequency adaptation mechanisms as follows:

$$
\dot{v}_{0}(t)=-\gamma v_{0}(t)+\lambda g\left(v_{i n}(t), v_{0}(t)\right)+n(t) .
$$

where $n(t)$ is white noise that models random fluctuations in ion currents [14], $\gamma$ is decay rate and $\lambda$ links firing rate to slow state. Thus the dynamics of $v_{0}$ is influenced by firing rates that in turn cause changes in excitability of the neural population, which may initiate seizures.

\section{Simulated $i E E G$}

Simulated iEEG using the (slow-fast) DNMM, $y_{k}$ (contaminated by measurement noise with Gaussian distribution, $r_{k}$, with zero mean and variance, $\sigma$ ) is obtained by sampling mean membrane potential of the pyramidal population

$$
y_{k}=v_{e}\left(t_{k}\right)-v_{i}\left(t_{k}\right)+r_{k}, \quad k=\{1,2, \ldots, n\}
$$

where $n$ is the number of samples. The DNMM in Figure 1 can be written as a continuous-discrete dynamical system:

$$
\begin{aligned}
& \dot{v}(t)=f_{\theta}(v(t))+n(t) \\
& y_{k}=y\left(t_{k}\right)=H v+r_{k}, \quad k=\{1,2, \ldots, n\},
\end{aligned}
$$

where $v=\left[v_{e}, v_{i}, v_{p}, v_{j}^{p}, v_{j}^{e}, v_{j}^{i}\right]^{\top}$ is states vector, $f$ is a nonlinear function denotes concatenated equations for dynamics of all neuronal populations, $\theta$ is a vector of constant model parameters (e.g, $\left.\alpha, v_{0}, \zeta\right), H=[1,-1,0,0,0,0]^{\top}$ is a constant vector, $y_{k}$ is a discrete observation, and $n(t)$ and $r_{k}$ are mutually uncorrelated endogenous (random neuronal fluctuations) and recording noise, respectively. In this paper, one of the aim is to infer parameter $\theta$, given time series of iEEG data, i.e., $y_{k}$.

\section{Model Identification}

A genetic algorithm (GA) is employed to find DNMM parameters that minimize a log likelihood function (LLF) of the model fitting a given iEEG data with paroxysmal transitions as illustrated in Figure 2. The GA creates parameters for which the cost function (i.e., LLF) is evaluated and ranked. Biologically inspired operations elite, crossover and mutation are then used to create new parameter sets (children) from better performing sets (parents). The LLF is the logarithm of 


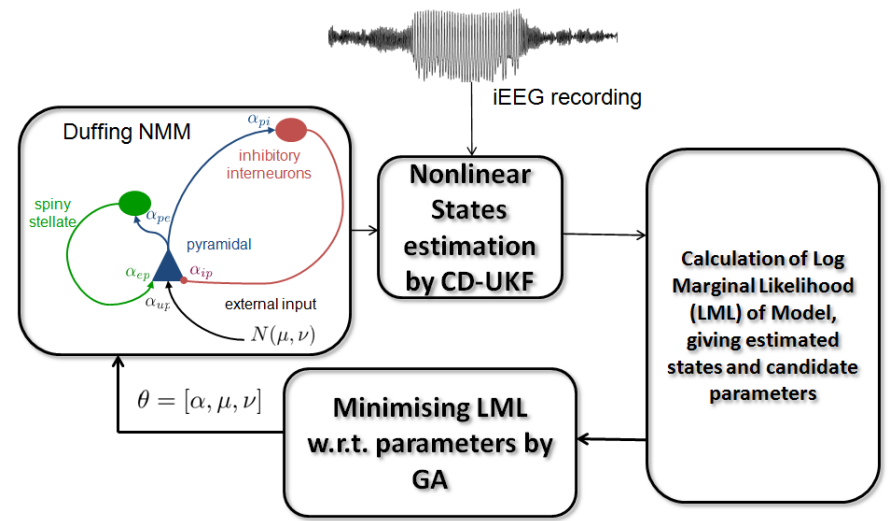

Fig. 2. DNMM parameter estimation method. A genetic algorithm is used to search parameter space and create instances of parameters. CD-UKF is employed to estimate hidden model states. The estimated states are in turn being used to calculate a log likelihood function (LLF). LLF acts as the cost function of GA to refine parameters until optimisation criteria is met and optimal parameters to model the data are found.

the product of conditional probability density functions(mean field approximation) for all recording samples in iEEG data, $(1: d)$, as follows:

$$
\begin{aligned}
\operatorname{LLF}\left(\theta \mid y_{1: d}\right) & =-\log \left(p\left(y_{1: d} \mid \theta\right)\right) \\
& =-\log \left(\left(\prod_{k=2}^{d} p\left(y_{k} \mid y_{k-1}, \theta\right)\right) p\left(y_{1} \mid \theta\right)\right) .
\end{aligned}
$$

Due to multi scale nature of the DNMM, the continuousdiscrete unscented Kalman filter (CD-UKF) is used for state estimation and approximation of the LLF. The (discrete) $\mathrm{UKF}$ is derivative-free Kalman filtering that approximates probability density functions for discrete states and observations by Gaussian distributions through unscented transforms [15]. The CD-UKF extends the discrete unscented Kalman filter (UKF) for continuous dynamical systems [15]. Using the CD-UKF algorithm, Eq. (8) can be written in terms of a product of Gaussian functions [16] as follows:

$$
\operatorname{LLF}\left(\theta \mid y_{1: d}\right)=-\log \left(\prod_{k=2}^{d} \frac{\exp \left(\frac{-1}{2} \varepsilon_{k}(\theta)^{\top} S_{k}^{-1}(\theta) \varepsilon_{k}(\theta)\right)}{\sqrt{\operatorname{det}\left(S_{k}(\theta)\right)(2 \pi)}}\right) .
$$

where $\varepsilon_{k}(\theta)=y_{k}-\mu_{k}(\theta)\left(\mu_{k}(\theta)\right.$ is the mean estimation of $\left.y_{k}\right)$, and $S_{k}(\theta)$ is the estimation covariance.

\section{RESULTS}

\section{A. Duffing Neural Mass Simulation}

The DNMM is simulated using the stochastic RungeKutta algorithm [17] on desktop computer and a result is shown in Figure 3. The model exhibits transitions to and from seizures resulting from random input to the model (i.e., if the model integrates with constant input it does not show switching dynamics). In particular, the model exhibits recurrent seizures, which is one of the features of epilepsy. Bifurcation analysis with respect to model input is carried out to study and compare the DNMM and JRNMM, shown in Figure 4. The DNMM has a wider range of unstable fixed points compared to the JRNMM due to the effect of

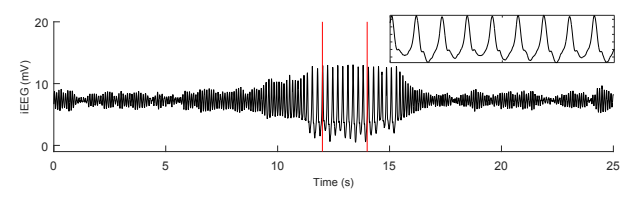

Fig. 3. Simulation of Duffing Neural Mass Model. The model exhibits apparently normal activity followed by transitions to and from seizure-like activity. The model is excited by random input with a Gaussian distribution.
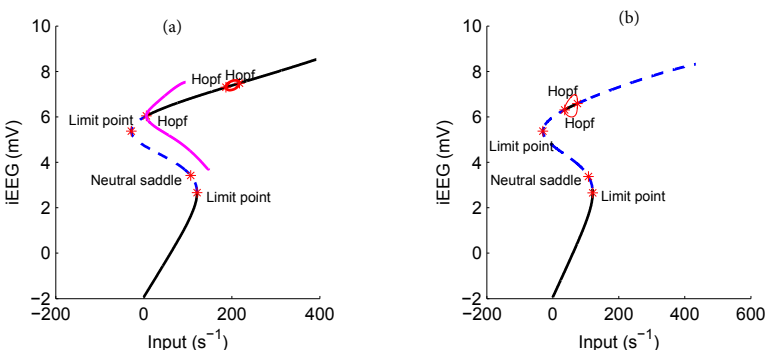

Fig. 4. Bifurcation Diagrams of the (a) Jansen-Rit NMM and (b) Duffing NMM. Comparing the two bifurcation diagrams shows that the DNMM has wide range of unstable fixed points compared to the JRNMM. Also, the JRNMM has a Hopf point (around input $50 s^{-1}$ ) that does not exist in the DNMM.

perturbation to the JRNMM that makes the synaptic transmission mechanisms sensitive to variations of their inputs. Continuation starting from limit point (LP) in the DNMM shows a wide range of spike-like limit cycles as well as fold bifurcation, shown in Figure 5. The co-existence of two dynamical regimes (seizure-like limit cycles and background states) in the model, together with contributions of the level of noise in the DNMM, makes this system prone to emulate transitions into and out of seizures.

\section{B. Slow-fast Duffing Neural Mass Model Simulation}

The slow-fast DNMM is simulated as shown in Figure 6, with firing threshold of the inhibitory population as the slow state. The simulated iEEG shows two types of seizures. The occurrence of the first seizure is due to random

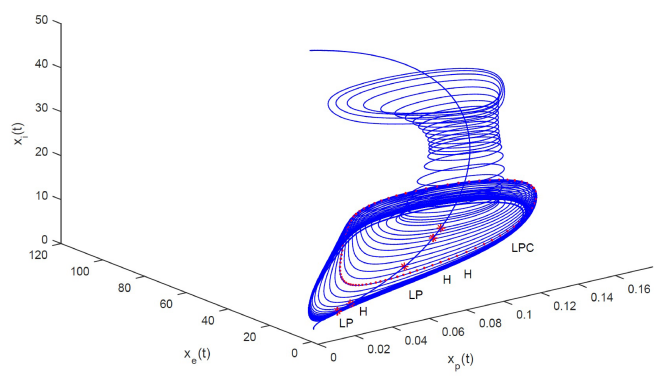

Fig. 5. Continuation of limit point (LP) in Duffing NMM with respect to input. There are limit cycles as well as folded bifurcation (limit point of cycle-LPC) points that co-exist with fixed points in the system. In addition, at LPC point, Homoclinic bifurcation is detected in Duffing NMM. The bifurcation properties in Duffing NMM make the system prone to switch back and forth between normal and seizures in the presence of noise. 

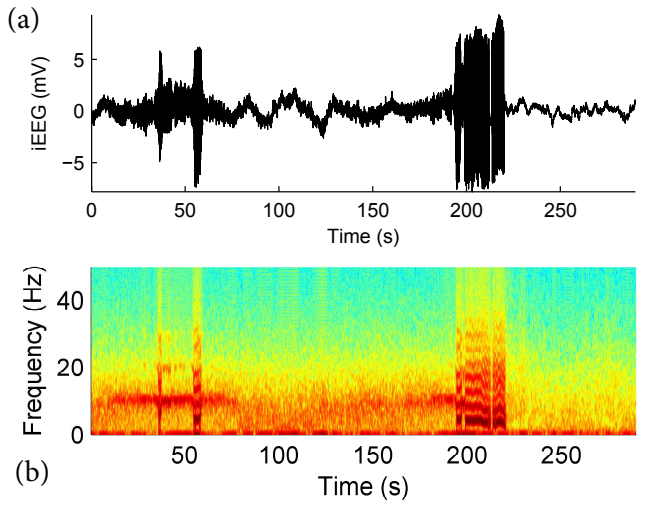

Fig. 6. Simulation of Slow-Fast Duffing NMM. (a) iEEG with paroxysmal transitions where the first seizure is due to Duffing terms in the model and the second seizure is due to slow dynamics of firing thresholds. (b) Spectrogram of iEEG shows frequency patterns seizures-like spikes and apparently normal activity.

TABLE I

PARAMETER ESTIMATION RESULTS.

\begin{tabular}{|c||c||c|}
\hline Parameter & Actual & Estimated \\
\hline$\alpha$ (Connectivity) & 136 & 135.60 \\
\hline$m$ (Mean of Input) & 191 & 189.50 \\
\hline$v$ (Variance of Input) & 1.20 & 1.24 \\
\hline
\end{tabular}

input to the model and of second one is related to slow dynamics of firing thresholds (this is verified by simulating the model with and without the Duffing terms in the model). A comparison between the post-ictal iEEG (in the second course of seizures) and pre-ictal activity may resemble lack of neuronal energy after seizures, which is observable in iEEG data from epileptic patients.

\section{Model Identification}

To illustrate the efficacy of the proposed identification method, iEEG data with paroxysmal transitions is generated using the DNMM. Then, three parameters (connectivity constants and input mean and variance) of the DNMM are considered as unknown and the GA-based identification method employed to recover these parameters. For each instance for parameters, the LLF is approximated by using the CD-UKF. Then optimization of the LLF is performed using a population size of 200 and the GA with mutation rate 0.3 and crossover rate 0.7 . The mutation rate 0.3 allows the GA to search in a wide range of search space for several generations and not become trapped in local minima. The criterion for which the GA terminates was when the average change in fitness values for 20 generations remained less than a tolerance $10^{-6}$. The results of applying the algorithm are promising and are given in Table 1 for an example run.

\section{CONCLUSIONS}

This paper introduced the DNMM, which can replicate stochastic generation of seizures. It also illustrated, by augmenting slow dynamics with this model, a complex pattern (either caused by slow dynamics of parameters and/or stochastic mechanisms) of transitions to and from paroxysmal activity similar to iEEG data. The dynamics of the slow-fast Duffing model suggests that underlying causes of seizures may change over time (as shown in Figure 6), which in turn may provide insights into why seizure prediction should be patient-specific. In general, using CD-UKF and GA for parameter estimation is time consuming. This is because, first, for each candidate parameters, CD-UKF should estimate the model states for all data points (which accordingly be used to calculate log likelihood function) and second, GA based optimisation, in general, requires many generation to find parameters that govern dynamics of data. However, the parameter identification based on optimization of the likelihood function showed promising performance, which can be applied to patient-specific modelling of iEEG recordings from epilepsy patients.

\section{REFERENCES}

[1] G. Baier, M. Goodfellow, P. N. Taylor, Y. Wang, and D. J. Garry. The importance of modelling epileptic seizure dynamics as spatio-temporal patterns. Frontiers in physiology, 3, 2012.

[2] B. Jansen, and V. Rit, Electroencephalogram and visual evoked potential generation in a mathematical model of coupled cortical columns, Biological Cybernetics, vol. 73, pp. 357-366, 1995.

[3] P. Suffczynski, F.L. da Silva, , J. Parra, D. Velis, S. Kalitzin, Epileptic transitions: model predictions and experimental validation. Journal of clinical neurophysiology, vol 22, pp.288-299,2005.

[4] S.N. Kalitzin, D.N. Velis, F.H.L da Silva, Stimulation-based anticipation and control of state transitions in the epileptic brain, Epilepsy \& Behavior,vol. 17, pp. 310-323, 2010.

[5] D. R. Freestone, D. Nesic, A. Jafarian, and D. B. Grayden, A neural mass model of spontaneous burst suppression and epileptic seizures, Annual International Conference of the IEEE Engineering in Medicine and Biology Society, 2013.

[6] V.K. Jirsa, W.C. Stacey, P.P. Quilichini, A.I. Ivanov, C. Bernard, On the nature of seizure dynamics, Brain, vol 137,pp 2210-2230, 2014.

[7] E.C. Zeeman, E.C, Duffing's Equation in Brain Modelling, University of Warwick, Mathematics Institute, 1975.

[8] R. Srebro,The Duffing oscillator: a model for the dynamics of the neuronal groups comprising the transient evoked potential, Electroencephalography and Clinical Neurophysiology/Evoked Potentials Section, vol. 96, pp 561-573, 1995.

[9] M. Toms-Rodrguez, S.P. Banks, Linear, time-varying approximations to nonlinear dynamical systems: with applications in control and optimization (Vol. 400). Springer Science \& Business Media, 2010.

[10] G. Ahmadi. Mean square response of a Duffing oscillator to a modulated white noise excitation by the generalized method of equivalent linearization. Journal of sound and Vibration, 71(1), pp.9-15.1980.

[11] P. A. Valdes, J. C. Jimenez, J. Riera, R. Biscay, T. Ozaki, Nonlinear EEG analysis based on a neural mass model,Biological Cybernetics, vol 81,pp 415-424, 1999.

[12] D.A. Prince, Neurophysiology of epilepsy. Annual review of neuroscience, vol 1(1), pp.395-415, 1978.

[13] J.A. Armijo, M. Shushtarian, E.M. Valdizan, A. Cuadrado, J. Adin, Ion channels and epilepsy. Current pharmaceutical design, 11(15), 19752003, 2005.

[14] C. Finke, J. Vollmer, S. Postnova, S., H.A. Braun, Propagation effects of current and conductance noise in a model neuron with subthreshold oscillations. Mathematical biosciences, 214(1-2), 109-121, (2008).

[15] S. Sarkka, On unscented Kalman filtering for state estimation of continuous-time nonlinear systems, IEEE Transactions on automatic control, Vol 52, Num 9, pp.1631-1641, 2007.

[16] J. Carlsson., C Nordheim, A parameter estimation method for continuous time dynamical systems based on the unscented Kalman filter and maximum likelihood, Chalmers University of Technology, 2011.

[17] J. Wilkie. Numerical methods for stochastic dierential equations. Physical Review E, 70 (1):017701, 2004. 